\title{
Decreasing the Duration of Point of Decision to Getting Non-stress Test Done: A Quality Improvement Study
}

\author{
Manisha Jhirwal ${ }^{1}$ (i) $\cdot$ Charu Sharma ${ }^{1} \cdot$ Shashank Shekhar ${ }^{1} \cdot$ Pratibha Singh $^{1} \cdot$ Satya Prakash Meena ${ }^{2}$
}

Received: 8 July 2021 / Accepted: 11 August 2021 / Published online: 2 October 2021

(c) Federation of Obstetric \& Gynecological Societies of India 2021

\begin{abstract}
Introduction Non-stress test is an important non-invasive tool of antepartum surveillance. The hypoxia, acidemia in the fetus can easily be picked up by a non-stress test. It is important to get a non-stress test done on time to prevent the adverse neonatal outcome.

Aim This quality improvement project aims to evaluate the waiting period for a non-stress test (NST) from the point of decision in the antenatal outpatient department and to increase the percentage of pregnant women getting NST done in less than $4 \mathrm{~h}$ from the point of decision from a baseline of $41 \%$ to $80 \%$ in 4 weeks.

Method This observational study was conducted in the Department of Obstetrics \& Gynecology at All India Institute of Medical Sciences Jodhpur for twelve weeks. This study has three phases-baseline assessment, implementation phase and continued implementation with the assessment phase. The tool used for the analysis of this problem was the Fishbone method. Results The baseline assessment showed that only $41 \%$ of pregnant women got the non-stress test done in $4 \mathrm{~h}$. We conducted a plan-do-study-act (PDSA) cycle 3 times, and the target was achieved in the second PDSA cycle.

Conclusion This quality improvement project has demonstrated that increasing awareness among all the team members who are dealing with pregnant women requiring NST can significantly increase the percentage of pregnant women getting NST done within $4 \mathrm{~h}$ from the point of decision. By detecting the fetal heart rate variation, we can prevent adverse fetal and neonatal outcomes.
\end{abstract}

Keywords Antepartum surveillance $\cdot$ Fetal hypoxia $\cdot$ Fishbone method $\cdot$ Non-stress test · Quality improvement

Dr. Manisha Jhirwal, Assistant Professor, Obstetrics \&

Gynecology, All India Institute of Medical Sciences, Jodhpur,

Rajasthan; Dr. Charu Sharma, Associate Professor, Department

of Obstetrics \& Gynecology, All India Institute of Medical

Sciences, Jodhpur, Rajasthan; Dr. Shashank Shekhar, Professor,

Department of Obstetrics \& Gynecology, All India Institute

of Medical Sciences, Jodhpur, Rajasthan; Dr Pratibha Singh,

Professor and Head of Department of Obstetrics \& Gynecology,

All India Institute of Medical Sciences, Jodhpur, Rajasthan;

Dr Satya Prakash Meena, Assistant Professor, Department of

General Surgery, All India Institute of Medical Sciences, Jodhpur,

Rajasthan.

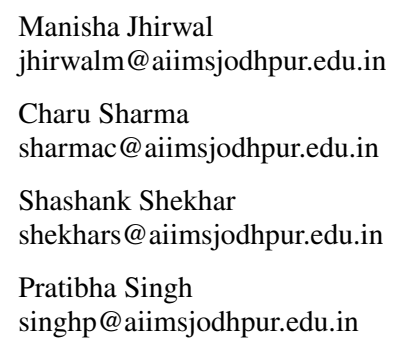

Manisha Jhirwal

jhirwalm@aiimsjodhpur.edu.in

Charu Sharma

sharmac@aiimsjodhpur.edu.in

Shashank Shekhar

shekhars@aiimsjodhpur.edu.in

Pratibha Singh

singhp@aiimsjodhpur.edu.in

Satya Prakash Meena

drsatyaprakash04@gmail.com

1 Department of Obstetrics \& Gynecology, All India Institute of Medical Sciences, Jodhpur, Rajasthan, India

2 Department of General Surgery, All India Institute of Medical Sciences, Jodhpur, Rajasthan, India 


\section{Introduction}

A prenatal non-stress test (NST) is the method to test the wellbeing of a fetus after 30 weeks of pregnancy and before the onset of labor. It is an important non-invasive tool of antepartum surveillance.

Hypoxemia or acidemia can alter the fetal heart rate pattern, fetal activity and degree of muscular tone. Due to hypoxemia, there is an alteration in redistribution in fetal blood leading to diminished renal flow and oligohydramnios. [1] The uteroplacental compromise can be picked up by surveillance techniques like a non-stress test, cardiotocography and real-time ultrasonography.

The principle of the non-stress test is based on the premise that in a non-acidotic or neurologically sound fetus, the heart sound accelerates with fetal movement. [2] Loss of this temporary acceleration signifies fetal sleep cycle or sometimes associated with depression of the central nervous system in the acidotic fetus. The NST can be initiated at the gestation age of 28 weeks, but it becomes reactive at 32 weeks' period of gestation. [3] It is not recommended to do NST routinely. There are few indications in which NST should be done. [4] (Table 1).

NST can be done in a semi-recline or supine position with a lateral tilt to prevent supine hypotension. It is conducted for a minimum period of $20 \mathrm{~min}$ but can be extended for $40 \mathrm{~min}$.

Table 1 Indications of prenatal NST

Indications

1. Post-term pregnancy

2. Decreased fetal movements

3. Oligohydramnios

4. Cholestasis of pregnancy

5. Multiple pregnancies

6. Hypertensive disorder in pregnancy (chronic HTN, pre-eclampsia)

7. Diabetes mellitus (Pregestational or gestational)

8. Other conditions-hypothyroidism, antiphospholipid syndrome, SLE
NST machine has two transducers. The doppler transducer is used to monitor the fetal heart sound, and the pressure transducer is used to monitor uterine contraction. On the tracing of NST, the number, amplitude and duration of acceleration in correlation with fetal movement are assessed.

NST is categorized into two: reactive or non-reactive. As per the American College of Obstetricians and Gynecologist (ACOG), a reactive NST has two or more accelerations peak at $15 \mathrm{bpm}$ or more above baseline, each lasting $15 \mathrm{~s}$ or more and all occurring within $20 \mathrm{~min}$ of beginning the test. The non-reactive NST has no acceleration in fetal heart rate over $40 \mathrm{~min}$. Non-reactive NST is seen normally in a fetal sleep cycle. If non-reactive NST has the deceleration persisting for one minute or more with no or less beat-to-beat variability, may increase the risk of fetal demise. [5] Therefore, in high-risk patients getting NST done on time becomes more important to prevent fetal demise.

According to National Institute of Health and Care Excellence (NICE) guidelines 2017, cardiotocography trace is categorize as normal (all features are reassuring), suspicious (one non-reassuring feature and two reassuring feature) and pathological (one abnormal feature and two non-reassuring feature). (Table 2).

For a baseline, we evaluated the waiting period for NST from the point of decision in the antenatal outpatient department (ANC OPD). This quality improvement project aims to evaluate the waiting period for a non-stress test (NST) from the point of decision in the antenatal outpatient department and to increase the percentage of pregnant women getting NST done in less than $4 \mathrm{~h}$ from the point of decision from a baseline of $40 \%$ to $80 \%$ in 4 weeks.

\section{Methodology}

This observational study was conducted in the Department of Obstetrics \& Gynecology at All India Institute of Medical Sciences Jodhpur for twelve weeks. This study was conducted in three phases-three weeks for baseline

Table 2 NICE classification of fetal heart rate trace features

\begin{tabular}{lllll}
\hline Features & Baseline FHR & Variability & Deceleration & Acceleration \\
\hline $\begin{array}{l}\text { Reassuring } \\
\text { Non-reassuring }\end{array}$ & $\begin{array}{l}110-160 \\
100-109 \\
\text { or } \\
161-180\end{array}$ & $\begin{array}{l}\text { More than } 5 \\
\text { Less than 5 for 40- 90 min }\end{array}$ & $\begin{array}{l}\text { None } \\
\text { Typical variable deceleration with } \\
\text { over 50\% of contraction, occurring } \\
\text { for over 90 min. Single prolonged } \\
\text { deceleration for up to 3 m }\end{array}$ & $\begin{array}{l}\text { The absence of acceleration with } \\
\text { otherwise normal trace is of } \\
\text { uncertain significance }\end{array}$ \\
Abnormal & $<100$ & Less than 5 for 90 min & $\begin{array}{l}\text { Either atypical variable } \\
\text { decelerations with over 50\% of } \\
\text { contractions or late decelerations, } \\
\text { both for over 30 min. Single } \\
\text { prolonged deceleration for more } \\
\text { than 3 min }\end{array}$ & $\begin{array}{l}\text { The absence of acceleration with } \\
\text { otherwise normal trace is of } \\
\text { uncertain significance }\end{array}$ \\
\end{tabular}


assessment, four weeks for implementation and five weeks for continued implementation and assessment phase.

It included all the pregnant women with gestational age more than 32 weeks attending ANC OPD on Monday, Wednesday and Friday requiring NST. The pregnant women who required immediate treatment for antepartum hemorrhage and intrauterine fetal death were excluded from the study. The time was noted when the patient was advised to get the NST done by a consultant or senior resident in ANC OPD.

\section{Baseline Assessment}

It has been observed that there was a long waiting time for the non-stress test to get done from the point of decision in ANC OPD, AIIMS Jodhpur. The pregnant women attending ANC OPD (Monday, Wednesday and Friday) were analyzed for three weeks. It included 12 pregnant women. The exact time of advising NST and the exact time of getting NST done was noted.
It was observed that only $41 \%$ of cases (5 out of 12 patients) got the NST done in $4 \mathrm{~h}$. The remaining 59\% (7 out of 12 patients) got NST done in 6 to $8 \mathrm{~h}$. Among these seven pregnant women, two pregnant women had suspicious NST. (Table 3) This long waiting time of NST can delay the decision-making in pregnant women and results in adverse fetal or neonatal outcomes.

We decided to find out the factors causing delay in getting NST done on time. Therefore, we could shorten the time required in decision-making in pregnant mothers and prevent adverse neonatal outcomes.

\section{Design}

The baseline measurement was taken over three weeks. The purpose of this quality improvement project was to re-audit the baseline measurements after the identification of obstacles in getting NST done on time.
Table 3 Clinical profile and outcome of pregnant women who exhibited abnormal NST

\begin{tabular}{lll}
\hline Parameters & Patient 1 & Patient 2 \\
\hline Age (years) & 32 & 28 \\
Gravida/parity & G3P1011 & G2P1001 \\
Indication of NST & Fetal growth restriction & Decreased fetal movement \\
Period of gestation & 36 weeks 3 days & 39 weeks 5 days \\
Intervention done & Induced with dinoprostone gel & Induced with dinoprostone gel \\
Mode of delivery & Cesarean section & Cesarean section \\
Indication & Thick meconium stained liquor & Thick meconium stained liquor \\
& & with fetal bradycardia \\
Fetal outcome & 7,8 & 6,7 \\
APGAR score & $2500 g m s$ & $3020 \mathrm{gms}$ \\
Birth weight & & \\
NICU admission & Kept in observation for 4 h & Kept for 36 h in view of tachypnea \\
\hline
\end{tabular}

\begin{tabular}{|l|l|}
\hline \multicolumn{1}{|c|}{ POLICY } \\
Some staff does not know the importance \\
of getting NST done on time
\end{tabular}$\quad \begin{aligned} & \text { PEOPLE } \\
& \text { PGs and Nurses are busy with } \\
& \text { laboring patients }\end{aligned}$

\section{PLACE}

There is no separate room or NST

machine for OPD patients
Pregnant women not getting NST in $4 \mathrm{hrs}$

Fig. 1 Fishbone method 
The tool used for the analysis of this problem was the Fishbone method. (Figure 1).

From the plan-do-study-act (PDSA) cycle, we realized that we need to make a team for the implementation and anticipation of the plan.

\section{Strategy}

\section{Implementation Phase}

\section{PDSA Cycle 1}

The main question was "does the distance between OPD complex and labor room have an effect on getting NST done on time". As we don't have the NST machine in the OPD complex. Only two NST machines are there in the labor room complex which is far from the OPD complex. We planned to assign a guard to guide the pregnant women to reach the labor room complex from the OPD block. In the labor room, we have a team of staff nurses. We planned to assign a nursing staff in receiving area to attend to the patient immediately and asked to inform the junior resident as soon as possible. The junior resident was asked to note down the time when the patient was received in the NST room and to get the NST done for the pregnant women.

The focused group discussion was done with nursing staff and the junior resident posted in the labor room on a biweekly basis. The discussion was based on the difficulties encountered by patients in getting NST done and possible solutions.

\section{PDSA Cycle 2}

After the initial implementation of the plan, the data were presented among the team members. It allowed us to find out the cause of a decline in the percentage of getting NST done within four hours of the decision.

It was observed that there were some difficulties at the nursing staff level in the labor room complex, they were already overburdened. They did not feel that it was their duty to get NST done for pregnant patients.

After discussion, it was explained that they are just supposed to put the right probe at the right place which will not take more than few minutes. This minimal support can help in improving achieving the target. Senior staff was asked to convey the message to the other junior staff also.

\section{Continued Implementation and Assessment}

Team members were motivated in each meeting. PDSA cycle was done and followed biweekly until $80 \%$ of the pregnant women got the NST done within $4 \mathrm{~h}$ of the time of decision.

\section{Action taken after interpretation of NST tracing}

When the NST was reactive, pregnant women were reassured and allowed to follow up in ANC OPD.

In case of non-reactive NST, it was continued for next $20 \mathrm{~min}$ to find out whether its due to prolonged sleep or hypoxia. If NST remains non-reactive even after $60 \mathrm{~min}$, the fetus underwent evaluation with modified biophysical profile (BBP). If modified BBP was abnormal then according to the period of gestation, associated maternal conditions and pregnancy-related complications, the decision of termination of pregnancy was taken.

\section{Multi-disciplinary Team}

This quality improvement initiative included the consultant in OPD, guard posted in OPD, nursing staff and junior residents posted in the labor room (Figure 2).

\section{Results}

\section{PDSA Cycle 1}

There was no significant difference in the percentage of getting NST done within four hours in comparison with

\section{Pregnant women with gestational age more than 32} weeks requiring NST

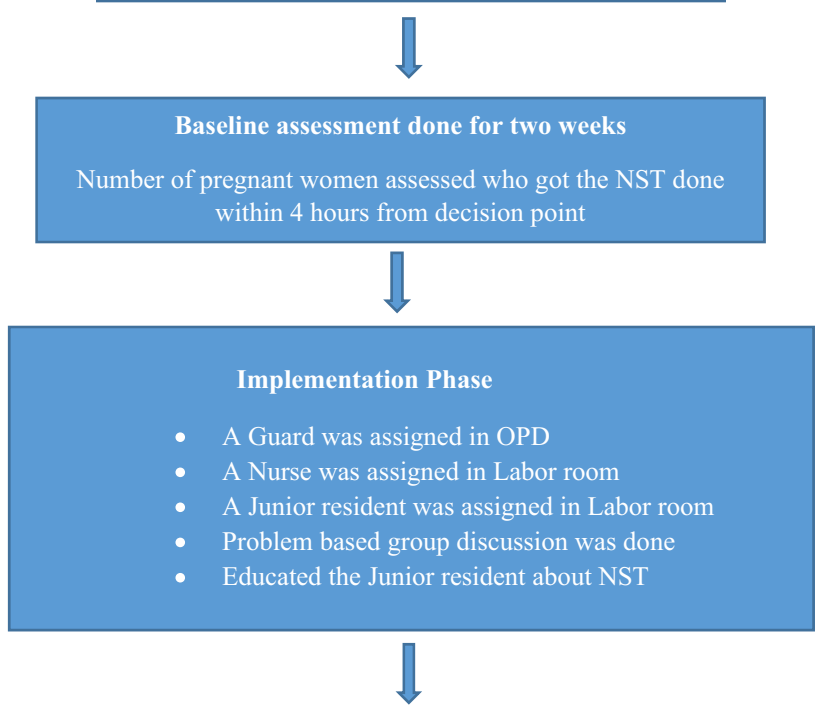

Continued Implementation and assessment Assessment done to look for increase in number of pregnant patients getting NST done within 4 hours from the point of decision.

Fig. 2 Multi-disciplinary team involved in the quality improvement project 
baseline measurements. In the initial phase of the implementation phase, 18 pregnant women were advised to get NST done but only eight pregnant women got that done in four hours $(44.4 \%)$.

Among these eight pregnant women getting NST done in less than $4 \mathrm{~h}$, only one pregnant women with complains of decreased fetal movement had pathological NST. Total 10 high-risk pregnant women got NST done after $6 \mathrm{~h}$. Out of these, three pregnant women had non-reactive NST. (Table 4) All these four patients underwent termination of pregnancy.

It indicated that the importance of getting NST done was not recognized widely among the labor room team. This lead to many pregnant women getting NST done after a long waiting of six hours. We carried out small educational sessions among the junior residents and senior residents posted in OPD and the labor room. The compliance of protocol was $46 \%$, and the number of pregnant women getting NST done was increased to $50 \%$.

\section{PDSA Cycle 2}

After regular meetings with team members biweekly, we implemented the plan again. After the implementation of the protocol, initially, there was a fall in the percentage of pregnant women getting NST done on time due to a change in the team in the labor room as we have rotational postings. We again conducted a small educational session among junior and senior residents regarding the protocol and importance of NST. In few days, there was an improvement in baseline measurements in which $80 \%$ of pregnant women got NST done with $4 \mathrm{~h}$ from the point of decision in OPD.

In four weeks of the implementation phase, the target of $80 \%$ (19 out of 22 pregnant women) was achieved.

\section{PDSA cycle 3}

Keeping in mind the findings of PDSA cycle 2, we continued the education session among the concerned team. The compliance was $80 \%$, and timely NST was done within $4 \mathrm{~h}$ in $90 \%$ of pregnant women.

\section{Discussion}

The fetal heart rate activity is thought to be a good indicator of normal fetal anatomical function. The non-stress test is a non-invasive test that can be used as a screening tool for the assessment of fetal wellbeing in high-risk pregnant women. It helps in the early detection of fetal compromise or risk of fetal death secondary to the high-risk pregnancy.

In the pregnancies complicated by pre-existing maternal condition or pregnancy-related complications, the non-stress test is widely used technique for antepartum fetal assessment The principle of NST is to recognize the coupling of fetal neurological status with cardiovascular reflexes. When there is fetal compromise, there will be disturbance or disappearance in one of the factor. Early detection and timely management of fetal complication can prevent maternal morbidity, stillbirth and neonatal morbidity and mortality.

Table 4 Clinical profile and outcome of pregnant women who exhibited abnormal NST after PDSA cycle one

\begin{tabular}{|c|c|c|c|c|}
\hline \multirow[b]{2}{*}{ Parameters } & \multirow{2}{*}{$\begin{array}{l}\text { Patient got NST done } \\
\text { in }<4 \mathrm{~h}(n=1) \\
\text { Patient } 1\end{array}$} & \multicolumn{3}{|c|}{ Patient got NST done in $>4 \mathrm{~h}(n=3)$} \\
\hline & & Patient 1 & Patient 2 & Patient 3 \\
\hline Age (years) & 28 & 24 & 32 & 29 \\
\hline Gravida/parity & G3P1001 & G1P0000 & G2P1001 & G3P0020 \\
\hline Indication of NST & $\begin{array}{l}\text { Decreased fetal movement } \\
\text { for two days }\end{array}$ & Fetal growth restriction & Pre-eclampsia & Overt diabetes on insulin \\
\hline Period of Gestation & 40 weeks 1 days & 34 weeks 0 days & 36 weeks 5 days & 35 weeks 1 days \\
\hline Intervention done & Emergency LSCS & $\begin{array}{l}\text { Induced with dinoprostone } \\
\text { gel }\end{array}$ & $\begin{array}{l}\text { Induced with dinoprostone } \\
\text { gel }\end{array}$ & Induced with dinoprostone gel \\
\hline Mode of Delivery & Cesarean section & Preterm vaginal delivery & Preterm vaginal delivery & Cesarean section \\
\hline Indication of LSCS & Fetal bradycardia & - & - & $\begin{array}{l}\text { Thick meconium stained liq- } \\
\text { uor with fetal bradycardia }\end{array}$ \\
\hline Fetal outcome & 5,6 & 7,8 & 7,8 & 6,7 \\
\hline $\begin{array}{l}\text { APGAR score } \\
\text { Birth weight }\end{array}$ & $2800 \mathrm{gms}$ & $1270 \mathrm{gms}$ & $2510 \mathrm{gms}$ & $2200 \mathrm{gms}$ \\
\hline NICU admission & $\begin{array}{l}\text { On continuous positive air- } \\
\text { way pressure for } 2 \text { days }\end{array}$ & $\begin{array}{l}\text { Kept for } 24 \mathrm{~h} \text { in view of } \\
\text { tachypnea }\end{array}$ & $\begin{array}{l}\text { Kept for } 24 \mathrm{~h} \text { in view of } \\
\text { tachypnea }\end{array}$ & $\begin{array}{l}\text { Kept for } 36 \mathrm{~h} \text { in view of hypo- } \\
\text { glycaemia }\end{array}$ \\
\hline
\end{tabular}


In present study, NST was recommended to women with high-risk pregnancy and decreased fetal movements in third trimester of pregnancy. Before this quality improvement project was conducted, many women were getting NST done in 5-8 $\mathrm{h}$ from the point of decision resulting in delay in termination of pregnancy leading to adverse perinatal outcome. In PDSA cycle 1, we had one patient who got NST done in less than $4 \mathrm{~h}$ and three patients who got NST done in more than $6 \mathrm{~h}$, respectively. All these patient had non-reactive NST. The decision of termination of pregnancy was taken according to the NST findings. If the intervention was not done on time, it could have led to adverse perinatal outcome. A study conducted by Raouf $\mathrm{S}$. et al. concluded that study group with non-reactive NST had more fetal complications like bradycardia, low APGAR score, stillbirth than those with reactive NST. Hence, NST is a valuable screening technique to be used routinely as a diagnostic test in prenatal period and latent phase of labor. [6] This quality improvement project helped in improving the quality of care given to antenatal patients. Our project aimed at increasing the percentage of pregnant women getting NST done in $4 \mathrm{~h}$ from 40.90 to $80 \%$. The aim was to get the NST done on time, detect the fetal compromise and manage the case timely to prevent adverse fetal or neonatal outcomes.

We observed multiple obstacles at various levels starting from the OPD complex to the labor room. Assigning a guard in ANC OPD helped the pregnant women approaching the labor room complex. We also put the footmarks from the OPD complex to the labor room complex to guide the direction.

In the labor room, we assigned particular nursing staff and junior residents to look after the pregnant patients, who needed the non-stress test done. We conducted fortnight meetings with the team involved in the project and discussed the problems with solutions.

The target was achieved in the second PDSA cycle only. But in the initial phase of implementation, there was a drop in the percentage of pregnant women who got NST done in $4 \mathrm{~h}$ due to the rotation of the medical team in the labor room and the OPD complex. The maintenance of these results required continuous motivation of all the team members like the guard in the ANC OPD, nursing staff and junior residents posted in the labor room complex.

This quality improvement project has demonstrated that simple measures like keeping a guard in ANC OPD, assigning a particular nursing staff in LR and teaching the junior resident about the importance of NST significantly increased the percentage of pregnant women getting NST done within $4 \mathrm{~h}$ from the point of decision. This is more likely due to increased awareness among junior residents and nursing staff about the importance of NST.

\section{Limitations}

Many lessons were learned during this quality improvement project. We encountered few problems like realising the need to keep watch on guard posted in ANC OPD, junior resident and nursing staff posted in LR. There were only two NST machines in the LR complex. When there are many laboring patients in LR, it was difficult to get NST done on the pregnant women sent from OPD.

For quality improvement, PDSA cycle and model for improvement need frequent data collection at short interval to find out the loop holes and change the strategy accordingly. Hence, auditing the large number of data can act as barrier in initiating improvements in the activity and filling the local gaps. Present study was conducted in the COVID19 era; hence, the sample size was small. However, the number of patients was enough to conduct the study and to assess the problems.

\section{Conclusion}

The NST is an important diagnostic tool for fetal assessment. Delay in getting NST done on time may result in adverse fetal or neonatal outcomes. This quality improvement project has demonstrated that increasing awareness among all the team members who are dealing with pregnant women requiring NST can significantly increase the percentage of pregnant women getting NST done within $4 \mathrm{~h}$ from the point of decision and avoid adverse perinatal outcome.

Funding None.

\section{Declarations}

Conflict of interest Authors declare no conflict of interest.

Informed consent was taken from all the patients.

\section{References}

1. Arnold K.C., Flint C.J. (2017) Antepartum Fetal Surveillance. In: Obstetrics Essentials. Springer, Cham. https://doi.org/10.1007/ 978-3-319-57675-6_28

2. Nazari S, Hatami E, TabatabaeiChehr M, Bagheri M, Ghorbani M. Diagnostic value of non-stress test interpreted by smart interpretive software. J Midwifery Reproduct Health. 2018;6(3):1384-9. 
3. Mirza N, Meena V, Garg R, Gupta V, Iqbal R, Meena K, Agarwal $\mathrm{N}$, Gupta S. Comparison of non stress test and umbilical artery doppler in high-risk pregnancy. Int J Med Sci Educ [Internet]. 2017Jul.1 [cited 2021Jul.8];4(2)

4. Eslamian ZL, Zarean E, Moshfeghi M, Heidari Z. Evaluation of the predictive value of fetal Doppler ultrasound for neonatal outcome from the 36th week of pregnancy. J Res Med Sci. 2018;20(23):13.

5. Wolf H, Arabin B, Lees CC, Oepkes D, Prefumo F, Thilaganathan B, et al. Longitudinal study of computerized cardiotocography in early fetal growth restriction. Ultrasound Obstet Gynecol. 2017;50(1):71-8.

6. Raouf S, Sheikhan F, Hassanpour S, Bani S, Torabi R, Shamsalizadeh N. Diagnostic value of non stress test in latent phase of labor and maternal and fetal outcomes. Glob J Health Sci. 2014;7(2):177-82.

Publisher's Note Springer Nature remains neutral with regard to jurisdictional claims in published maps and institutional affiliations.

\section{About the Author}

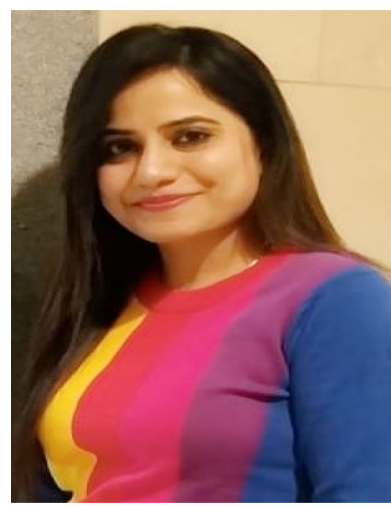

Manisha Jhirwal is an Assistan Professor in Department of Obstetrics \& Gynecology, AIIMS Jodhpur, Rajasthan. She completed her post-graduation from KGMU Lucknow in 2014. She has done fellowship in minimal access surgery and trained in USG obstetrics from ISUOG. She has keen interest in obstetrical critical care, laparoscopy and infertility. 